\title{
Bevacizumab Associated with Chemotherapy for Initially Non-resectable Liver Metastases from Colorectal Cancer: a Case-Control Study
}

\author{
MARIE ALT ${ }^{1}$, STÉPHANIE TRUANT ${ }^{2}$, THIBAUT RENAUD-VANTROYS ${ }^{1}$, \\ CÉDRIC SÉQUIER $^{2}$, ALAIN DUHAMEL ${ }^{3}$, MEHDI EL AMRANY ${ }^{2}$, ANTHONY TURPIN ${ }^{1}$, \\ SAMIRA MAKHLOUFI ${ }^{1}$, FRANÇOIS RENÉ PRUVOT $^{2}$ and MOHAMED HEBBAR ${ }^{1}$ \\ Departments of ${ }^{1}$ Medical Oncology, and ${ }^{2}$ Digestive Surgery and Transplantation, \\ ${ }^{3}$ Biostatistics; University Hospital, Lille, France
}

\begin{abstract}
Background: The place of bevacizumab in therapy of patients with initially non-resectable liver metastases from colorectal cancer (CRC) remains debated. Bevacizumab may increase the efficacy of chemotherapy but it may also maintain dormant micrometastases in a dormant state, eventually increasing the long-term likelihood of tumor relapse. The aim of this study was to explore this hypothesis. Patients and Methods: A retrospective case-control study was performed in patients with initially non-resectable CRC liver metastases. Metastases were rendered resectable after chemotherapy with (cases) or without (controls) bevacizumab. Cases and controls were matched for synchronicity, number and maximal size of metastases. The main objective was the disease-free survival (DFS). Results: A total of 82 patients were enrolled (41 in each group). The median DFS was 12.0 months in the bevacizumab group, and 10.2 months in the group treated with chemotherapy alone $(p=0.26)$. Conclusion: We observed no significant effect on DFS for bevacizumab when added to chemotherapy in patients with initially non-resectable liver metastases. Prospective trials on this issue are warranted.
\end{abstract}

Colorectal cancer (CRC) is the second cause of cancerrelated death in Western countries (1). Nearly half of all patients with CRC will develop metastases, the liver being the main metastatic site (2). Surgical resection is associated with a prolonged survival (40-50\% 5-year survival rate), with a hope for cure (3). Liver metastases are initially

Correspondence to: Pr. Mohamed HEBBAR, MD, Ph.D., Service d'Oncologie Médicale, Centre Hospitalo-Universitaire, 1 rue Michel Polonovski, 59037 Lille, France. Tel: +33 320445461, Fax: +33 320445023, e-mail: mohamed.hebbar@chru-lille.fr

Key Words: Bevacizumab, colorectal cancer, liver metastases. resectable in 15-20\% of cases, and a perioperative or postoperative FOLFOX [oxaliplatin, 5-fluorouracil (5-FU) and leucovorin] regimen is generally proposed (4). In the majority of patients, liver metastases are not initially resectable, but a chemotherapy (oxaliplatin/irinotecan-based regimen) can render these metastases resectable in 20-30\% of cases (5). Generally, after resection of initially nonresectable metastases, the same chemotherapy schedule is resumed after surgery. Currently, first-line chemotherapy in patients with non-resectable metastases is frequently associated with bevacizumab, an anti-antiogenic monoclonal antibody directed against vascular endothelial growth factor A (VEGFA). This strategy is based on the demonstrated benefit in terms of overall survival (OS) when bevacizumab was added to chemotherapy in a palliative setting (6).

The accurate place of bevacizumab in patients with liver metastases that are initially non-resectable but potentially resectable after conversion chemotherapy is still debated. Firstly, there are no available results from prospective randomized trials comparing chemotherapy alone to chemotherapy plus bevacizumab in this setting. Secondly, the increase in the objective response rate is only $10 \%$ by adding bevacizumab, which probably translates into a limited increase in the resection rate. Additionally, results of trials performed after resection of stage III colonic cancer indicated no benefit for use of bevacizumab in addition to oxaliplatin-based adjuvant chemotherapy $(7,8)$. Moreover, in the AVANT trial assessing oxaliplatin-based chemotherapy with or without bevacizumab after resection of a stage III colon cancer, a deleterious effect of bevacizumab for disease-free survival (DFS) was suggested (8). The explanation for this potent long-term deleterious effect remains unclear. One hypothesis is that bevacizumab may maintain residual dormant tumor cells in dormancy, a state in which these cells are resistant to chemotherapy. Escape from the dormant state is indeed related to the angiogenic 
Table I. Patients' baseline characteristics.

\begin{tabular}{lcc}
\hline Characteristic & Chemotherapy plus bevacizumab $(\mathrm{n}=41)$ & Chemotherapy alone $(\mathrm{n}=41)$ \\
\hline Median age (range), years & $60(39-79)$ & $65(28-84)$ \\
Male gender, $\mathrm{n}(\%)$ & $18(43.9)$ & $21(51.5)$ \\
Primary colon, $\mathrm{n}(\%)$ & $36(87.8)$ & $33(80.5)$ \\
Primary T stage, $\mathrm{n}(\%)$ & $3(7.3)$ & $4(9.7)$ \\
T1-T2 & $38(92.7)$ & $37(90.3)$ \\
T3-T4 & & $13(31.7)$ \\
Primary N stage, $\mathrm{n}(\%)$ & $13(31.7)$ & $24(58.6)$ \\
N0 & $27(65.8)$ & $4(9.7)$ \\
N+ & $1(2.5)$ & $23(56.1)$ \\
Nx & $33(80.5)$ & $3(1-10)$ \\
Bilobar liver metastases, $n(\%)$ & $3(1-10)$ & $4(1-14)$ \\
Median no. of metastases (range) & $4(1-13)$ & $31(75.6)$ \\
Median maximal diameter of metastases (range), $\mathrm{cm}$ & $31(75.6)$ & \\
Synchronous metastases, $\mathrm{n}(\%)$ & & $20(49.5)$ \\
Chemotherapy regimen, $\mathrm{n}(\%)$ & $3(7.3)$ & $21(51.5)$ \\
FOLFOX & $38(92.7)$ & $6(3-12)$ \\
FOLFIRI & $6(2-14)$ & $6(1-11)$ \\
Median no. of preoperative cycles (range) & $6(2-8)$ & \\
Median no. of postoperative cycles (range) & & \\
\hline
\end{tabular}

switch, with a prominent role of VEGFA (9). It is therefore theoretically possible that the addition of bevacizumab to chemotherapy in patients with initially non-resectable liver metastases will be associated with an increased risk of relapse.

In order to explore this hypothesis, we conducted a retrospective case-control study to assess the role of bevacizumab in such a setting.

\section{Patients and Methods}

We conducted a retrospective study performed on patients with initially non-resectable liver metastases from CRC in whom metastases were rendered resectable after chemotherapy with or without bevacizumab. All patients were treated at the University Hospital of Lille, France. The study has been declared to the local Ethic Committee.

Study population. Eligible patients were 18 years or older, with histologically confirmed CRC and initially unresectable liver metastases. The unresecability was stated by a multidisciplinary board including at least one experienced hepato-biliary surgeon. Patients were included if they had undergone a resection for metastases after conversion chemotherapy, with or without bevacizumab. Other eligibility criteria were as follows: no evidence of extra-hepatic metastases on computed tomographic scan and positon-emission tomography (PET). No previous chemotherapy in the metastatic setting was allowed.

Patients meeting the following criteria were excluded: macroscopic incomplete resection of metastases (R2), other cancer within 5 years (except for squamous cell carcinoma of the skin and in situ cervical cancer).
This was a case-control study, cases corresponding to patients who received chemotherapy plus bevacizumab, and controls corresponding to patients who received chemotherapy alone. In order to reduce selection bias, cases and controls were treated by the same multidisciplinary team at two different periods of time. The cases were treated between 2006 and 2010, after the approval of bevacizumab in first-line treatment of metastatic CRC (in 2005). The controls were treated between 2003 and 2005, when bevacizumab was not available. Using the same treatment period for cases and controls would have resulted in an imbalance between the two groups, very high-risk patients would probably have preferentially received chemotherapy plus bevacizumab.

Cases and controls were matched according to the main prognostic factors integrated by Fong et al.'s score (10): synchronicity of metastases (interval between primary and metastases, \pm 1 month); number of metastases (in the case of more than five metastases, patients were matched with a variability of \pm 2 units); maximal diameter of the metastases (in case of $>5 \mathrm{~cm}$ size, $\pm 1 \mathrm{~cm}$ variability was accepted).

Treatments. All patients received chemotherapy with or without bevacizumab every 2 weeks. FOLFOX regimen was defined as 85 $\mathrm{mg} / \mathrm{m}^{2}$ oxaliplatin intravenously on day 1 and $200 \mathrm{mg} / \mathrm{m}^{2}$ leucovorin intravenously on days 1 and $2,400 \mathrm{mg} / \mathrm{m}^{2}$ bolus of 5FU intravenously on day 1 followed by a 46-h infusion of fluorouracil $\left(2400 \mathrm{mg} / \mathrm{m}^{2}\right)$. FOLFIRI was defined as a $90-\mathrm{min}$ intravenous infusion of irinotecan $\left(180 \mathrm{mg} / \mathrm{m}^{2}\right)$ followed by a simplified LV5FU2 regimen $\left[400 \mathrm{mg} / \mathrm{m}^{2}\right.$ leucovorin and bolus fluorouracil $\left(400 \mathrm{mg} / \mathrm{m}^{2}\right)$ on day 1 followed by a $46-\mathrm{h}$ infusion of fluorouracil $\left.\left(2400 \mathrm{mg} / \mathrm{m}^{2}\right)\right]$. FOLFOX or FOLFIRI were administered with $5 \mathrm{mg} / \mathrm{kg}$ bevacizumab for cases.

Follow-up. Patients underwent a complete physical examination, imaging, and serum carcino-embryonic antigen (CEA) measurement, 
before initiation of the chemotherapy. Imaging was performed every four chemotherapy cycles, immediately before and within 4 weeks after surgery of metastases. A PET scan was performed before chemotherapy and before surgery. Imaging and CEA determination were repeated at the end of postoperative chemotherapy, every 3 months for 2 years, and every 6 months thereafter. The total number of chemotherapy cycles (with or without bevacizumab) was not fixed but our policy was to recommend six cycles before and six cycles after surgery.

Data collection. The following data were collected: demographic features: age and gender; primary tumor features: date of diagnosis, location (colon or rectum), date of resection, TNM status, and adjuvant chemotherapy; features of liver metastases: date of diagnosis, number of metastases, maximal size and localization inside the liver (one or two lobes); type of resection: R0 (microscopic negative margins) or R1 (microscopic positive margins); chemotherapy features: type of chemotherapy regimen, bevacizumab or not, total number of cycles, preoperative and postoperative number of cycles; follow-up: date of the last visit, date of recurrence, and date of death.

Objectives. The main objective was the DFS, defined as the interval between the resection of liver metastases and recurrence, regardless of the location, or death from any cause.

The OS was not retained as a main objective because, as mentioned above, cases and controls were not treated during the same periods of time. A patient in the control group treated before 2005 and experiencing relapse early could not receive targeted therapies [bevacizumab/anti-epidermal growth factor (EGFR)], in contrast to patients in the case group who were all treated after 2005. Consequently it was hazardous to compare OS after relapse. Hence OS was a secondary objective.

Statistical analysis. Statistical analyses were carried out with SAS (SAS Institute Inc., Cary, NC, USA). Patient baseline characteristics and disease were summarized using descriptive statistics. The link between the kind of treatment and DFS was studied by Cox's model (multivariate analysis). We used a fragility model to take into account the matching. In this model, the unpredictable effect was the block of matching. A value of $p \leq 0.05$ was considered statistically significant in all analyses. In this retrospective exploratory study, no required sample size calculation was performed.

\section{Results}

Patient's baseline characteristics. From January 2000 to May 2010, 82 patients were included: 41 patients in the chemotherapy alone group (controls) and 41 in the chemotherapy plus bevacizumab group (cases). Patient's characteristics were well balanced between the two groups (Table I).

Objective response and resection rate for liver metastases. The median number of chemotherapy cycles before surgery was 6 (range=2-14) in the bevacizumab group [data missing for two patients (6.5\%)] and $6(3-12)$ in the group treated with chemotherapy alone [data missing for three patients $(9.6 \%)$ ].

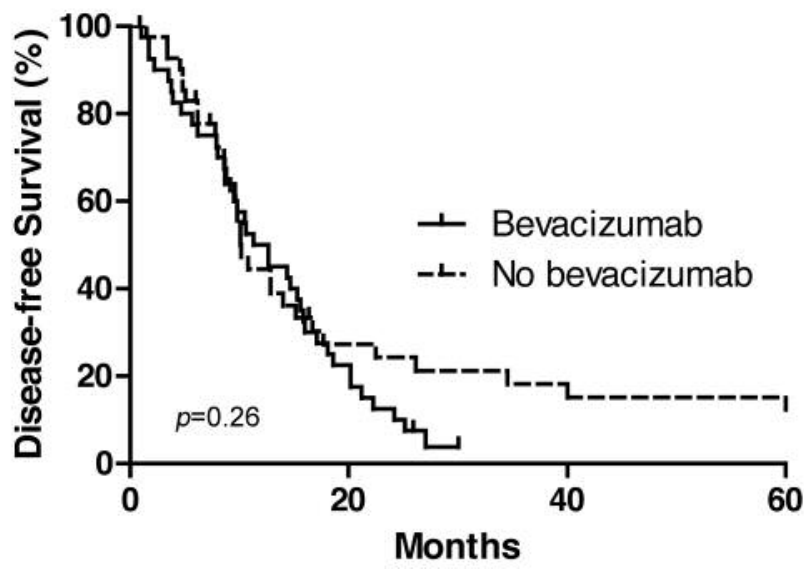

Figure 1. Disease-free survival after resection of liver metastases in patients who received chemotherapy plus bevacizumab $(n=41)$ or chemotherapy alone $(n=41)$.

Objective response to preoperative chemotherapy was obtained in 18 patients $(43.9 \%)$ in the bevacizumab group and 15 patients $(36.6 \%)$ in the group treated with chemotherapy alone. All other patients had a minimum response allowing resection of metastases. The resection types (R0 or R1) were similar between the two groups.

Disease-free survival. The median (range) DFS was 12.0 (0.9-30.0) months in the bevacizumab group, and 10.2 months (1.5-121.1) in the chemotherapy-alone group (Figure 1). This difference was not statistically significant [hazard ratio $(\mathrm{HR})=1.3,95 \%$ confidence interval $(\mathrm{CI})=0.81-2.15$; $p=0.26]$.

The median (range) OS was 31 (0.8-69.1) months and 43 (5.8-128.7) months in the bevacizumab group and the chemotherapy-alone group, respectively. This difference was not statistically significant $(\mathrm{HR}=1.0,95 \% \mathrm{CI}=0.23-5.0 ; p=0.92)$.

Synchronicity of metastases had no prognostic value for DFS $(\mathrm{HR}=0.5,95 \% \mathrm{CI}=0.31-1.08 ; p=0.08)$, as well as number of metastases $(\mathrm{HR}=1.0,95 \% \quad \mathrm{CI}=0$. 92-1.13; $p=0.65)$. Maximal size of metastases was a prognostic factor for DFS $(\mathrm{HR}=1.0,95 \% \mathrm{CI}=1.009-1.184 ; p=0.02)$.

\section{Discussion}

The question of whether to add a targeted therapy to chemotherapy in patients with potentially resectable liver metastases from CRC remains crucial. The present case-control study showed no benefit in terms of DFS when bevacizumab was added to conversion FOLFOX or FOLFIRI chemotherapy. We even observed that the survival curves tended to separate long-term in favor of the chemotherapy-alone group. These results should be interpreted cautiously, regarding the 
retrospective nature of the study, and the small sample size. Moreover, in order to avoid selection bias, we had to include cases and controls treated in two different periods of time. Controls were operated on before the registration of bevacizumab, and most of them before registration of antibodies to EGFR. Consequently, no comparison could be performed in terms of OS. Yet the two groups were well balanced for baseline features, especially tumoral characteristics. We used the main criteria of the score of Fong et al., i.e. number, maximal size and synchronicity of metastases. We did not retain the two remaining criteria of this score, nodal status of the primary and preoperative CEA concentration. In fact the distributions of nodal status were not significantly different between the two groups. CEA concentration was not available for all patients, and its prognostic value remains debated $(11,12)$. Finally, the prognostic score of Fong et al. was established in patients with resected metastases who received no chemotherapy or 5-FU monotherapy before the era of oxaliplatin and irinotecan. In the recent MIROX study performed on 284 patients with initially resectable metastases, we compared two chemotherapy schedules: 12 FOLFOX4 cycles vs. six FOLFOX7 cycles followed by six FOLFIRI cycles. The chemotherapy timing was decided by the investigator and was either perioperative or postoperative. All patients were stratified for the chemotherapy timing and Fong et al.'s score. No difference was found in terms of DFS and OS. Interestingly, the multivariate analysis indicated a prognostic value only for number, size, and synchronicity of metastases (13).

Of note, in the present study, more patients in the bevacizumab group had bilobar metastases, but in a recent retrospective study on 273 patients operated on for liver metastases, we found no prognostic value for distribution of liver metastases (14).

Another theoretical criticism regarding the present study is that all selected patients were operated on, which does not allow assessment of the potent impact of bevacizumab on resection rate. Our study was rather designed to assess the long-term effects of bevacizumab on DFS in patients who had a successful surgical procedure. Moreover, the impact of bevacizumab on resection rate of metastases remains debated, and has not been directly assessed in prospective trials comparing chemotherapy with or without bevacizumab in patients with metastases limited to the liver.

Two recent trials assessed the place of bevacizumab in patients with initially non-resectable metastases, but bevacizumab was used in all investigational arms. The randomized phase II OLIVIA trial compared bevacizumab plus FOLFOX and bevacizumab plus FOLFOXIRI in 80 patients with non-resectable liver metastases (15). Bevacizumab-FOLFOXIRI appeared superior in terms of the R0 resection rate $(48.8 \%$ vs. $23.1 \%, p=0.017)$ and in terms of DFS (18.8 vs. 12.0 months). Yet the specific place of bevacizumab cannot be determined from this trial.
The phase III TRIBE trial compared bevacizumab plus FOLFIRI and bevacizumab plus FOLFOXIRI in 508 patients with non-resectable liver metastases (16). No significant difference was observed between the two arms, but the quadritherapy appeared superior in terms of progression-free survival (12.1 vs. 9.7 months, $p=0.003$ ). Once again, the clear place of bevacizumab was not assessable.

A recent retrospective study evaluated the impact of bevacizumab added to perioperative chemotherapy on survival in 501 patients (17). In this study 384 patients were compared who received chemotherapy (generally FOLFOX) plus bevacizumab, and 117 patients who received chemotherapy alone. Bevacizumab had no effect on 3-year DFS rate $7.4 \mathrm{vs}$. $7.9 \%, p=0.082)$ and OS rate (76.4 vs.79.8\%). Regarding DFS, the curve for patients treated with chemotherapy alone was above that for the chemotherapy plus bevacizumab group. This study was performed on a large patient series but the patients were not matched regarding the main prognostic factors. We cannot exclude that patients who received bevacizumab had a more aggressive disease. Specifically, a trend for imbalance appeared for the number of metastases $(p=0.072)$. Additionally, discrepancies were observed for follow-up between the two arms, explained by the fact that only patients followed-up for at least 12 months were included. Nonetheless, and interestingly, the results were similar to those of our study.

Another targeted therapy, cetuximab, an antibody against EGFR, has been assessed in patients with initially resectable liver metastases from Kirsten RAS (KRAS) wild-type CRC in the New EPOC trial (18). This trial compared perioperative chemotherapy (generally FOLFOX) with or without cetuximab. The trial was stopped prematurely, with an apparent deleterious effect of cetuximab in terms of DFS (14.1 vs. 20.5 months). This result is consistent with those obtained in an adjuvant setting after resection of a stage III colon cancer (19). We cannot exclude a role for anti-EGFR therapy in patients with initially non-resectable liver metastases, taking into account that this setting has never been directly explored in prospective trials, similarly to bevacizumab.

Prospective trials evaluating bevacizumab and anti-EGFR are currently ongoing. The EORTC BOS-2 trial is comparing perioperative FOLFOX alone or associated with bevacizumab or panitumumab in patients with initially resectable liver metastases from RAS-wild type CRC, integrating the new KRAS and the NRAS mutations (20).

To conclude, our study indicated no significant effect on DFS from bevacizumab when added to perioperative chemotherapy in patients with initially non-resectable liver metastases from CRC. Prospective trials of bevacizumab in this setting are warranted for clarification of this issue.

\section{Clinical Practice Points}

The place of bevacizumab in addition to chemotherapy in patients with initially unresectable liver metastases from CRC remains 
controversial. In this retrospective case-control study, we found no benefit for adding bevacizumab in terms of disease-free survival.

Prospective trials of bevacizumab in this setting are warranted for clarification of this issue.

\section{References}

1 Jemal A, Bray F, Center MM, Ferlay J, Ward E and Forman D: Global cancer statistics. CA Cancer J Clin 61: 69-90, 1971.

2 Weiss L, Grundmann E, Torhorst J, Hartveit F, Moberg I, Eder M, Fenoglio-Preiser CM, Napier J, Horne CH and Lopez MJ: Haematogenous metastatic patterns in colonic carcinoma: an analysis of 1541 necropsies. J Pathol 150: 195-203, 1986.

3 Fernandez FG, Drebin JA, Linehan DC, Dehdashti F, Siegel BA and Strasberg SM: Five-year survival after resection of hepatic metastases from colorectal cancer in patients screened by positron emission tomography with F-18 fluorodeoxyglucose (FDG-PET). Ann Surg 240: 438-447; discussion 447-450, 2004.

4 Nordlinger B, Sorbye H, Glimelius B, Poston GJ, Schlag PM, Rougier P, Bechstein WO, Primrose JN, Walpole ET, FinchJones M, Jaeck D, Mirza D, Parks RW, Mauer M, Tanis E, Van Cutsem E, Scheithauer W and Gruenberger T: Perioperative FOLFOX 4 chemotherapy and surgery versus surgery alone for resectable liver metastases from colorectal cancer (EORTC 40983): long-term results of a randomised, controlled, phase 3 trial. Lancet Oncol 14: 1208-1215, 2013.

5 Hebbar M, Pruvot F-R, Romano O and de Gramont A: Integration of neoadjuvant and adjuvant chemotherapy in patients with resectable liver metastases from colorectal cancer. Cancer Treat Rev 35: 668-675, 2009.

6 Hurwitz H, Fehrenbacher L, Novotny W, Cartwright T, Hainsworth J, Heim W, Berlin J, Baron A, Griffing S, Holmgren E, Ferrara N, Fyfe G, Rogers B, Ross R and Kabbinavar F: Bevacizumab plus irinotecan, fluorouracil, and leucovorin for metastatic colorectal cancer. N Engl J Med 350: 2335-2342, 2004.

7 Allegra CJ, Yothers G, O'Connell MJ, Kuebler JP, Colangelo LH, Petrelli NJ and Wolmark N: Bevacizumab in stage II-III colon cancer: 5-year update of the National Surgical Adjuvant Breast and Bowel Project C-08 trial. J Clin Oncol 31: 359-364, 2013.

8 De Gramont A, Van Cutsem E, Schmoll HJ, Tabernero J, Clarke S, Moore MJ, Cunningham D, Cartwright TH, Hecht JR, Rivera F, Im SA, Bodoky G, Salazar R, Maindrault-Goebel F, ShachamShmueli E, Bajetta E, Makrutzki M, Shang A, André T and Hoff PM: Bevacizumab plus oxaliplatin-based chemotherapy as adjuvant treatment for colon cancer (AVANT): a phase 3 randomised controlled trial. Lancet Oncol 13: 1225-1233, 2012.

9 Páez D, Labonte MJ, Bohanes P, Zhang W, Benhanim L, Ning Y, Wakatsuki T, Loupakis F and Lenz HJ: Cancer dormancy: a model of early dissemination and late cancer recurrence. Clin Cancer Res Off J Am Assoc Cancer Res 18: 645-653, 2012.

10 Fong Y, Fortner J, Sun RL, Brennan MF and Blumgart LH: Clinical score for predicting recurrence after hepatic resection for metastatic colorectal cancer: analysis of 1001 consecutive cases. Ann Surg 230: 309-318, 1999.

11 Sun LC, Chu KS, Cheng SC, Lu CY, Kuo CH, Hsieh JS, Shih YL, Chang SJ and Wang JY: Preoperative serum carcinoembryonic antigen, albumin and age are supplementary to UICC staging systems in predicting survival for colorectal cancer patients undergoing surgical treatment. BMC Cancer 9: 288, 2009.
12 Lin JK, Lin CC, Yang SH, Wang HS, Jiang JK, Lan YT, Lin TC, Li AF, Chen WS and Chang SC: Early postoperative CEA level is a better prognostic indicator than is preoperative CEA level in predicting prognosis of patients with curable colorectal cancer. Int J Colorectal Dis 26: 1135-1141, 2011.

13 Hebbar M, Chibaudel B, André T, Mineur L, Smith D, Louvet C, Dutel JL, Ychou M, Legoux JL, Mabro M, Faroux R, Auby D, Brusquant D, Khalil A, Truant S, Hadengue A, Dalban C, Gayet B, Paye F, Pruvot FR, Bonnetain F and de Gramont A: FOLFOX4 versus sequential dose-dense FOLFOX7 followed by FOLFIRI in patients with resectable metastatic colorectal cancer (MIROX): a pragmatic approach to chemotherapy timing with perioperative or postoperative chemotherapy from an open-label, randomized phase III trial. Ann Oncol 26: 340-347, 2015.

14 Truant S, Séquier C, Leteurtre E, Boleslawski E, Elamrani M, Huet G, Duhamel A, Hebbar M and Pruvot FR: Tumour biology of colorectal liver metastasis is a more important factor in survival than surgical margin clearance in the era of modern chemotherapy regimens. HPB 17: 176-184, 2015.

15 Gruenberger T, Bridgewater J, Chau I, García Alfonso P, Rivoire M, Mudan S, Lasserre S, Hermann F, Waterkamp D and Adam $\mathrm{R}$ : Bevacizumab plus mFOLFOX-6 or FOLFOXIRI in patients with initially unresectable liver metastases from colorectal cancer: the OLIVIA multinational randomised phase II trial. Ann Oncol 26: 702-708, 2015.

16 Loupakis F, Cremolini C, Masi G, Lonardi S, Zagonel V, Salvatore L, Cortesi E, Tomasello G, Ronzoni M, Spadi R, Zaniboni A, Tonini G, Buonadonna A, Amoroso D, Chiara S, Carlomagno C, Boni C, Allegrini G, Boni L and Falcone A: Initial therapy with FOLFOXIRI and bevacizumab for metastatic colorectal cancer. N Engl J Med 371: 1609-1618, 2014.

17 Rong Z, Martel G, Vandenbroucke-Menu F, Adam R and Lapointe R: Impact of peri-operative bevacizumab on survival in patients with resected colorectal liver metastases: an analysis of the LiverMetSurvey. HPB 16: 342-349, 2014.

18 Primrose J, Falk S, Finch-Jones M, Valle J, O'Reilly D, Siriwardena A, Hornbuckle J, Peterson M, Rees M, Iveson T, Hickish T, Butler R, Stanton L, Dixon E, Little L, Bowers M, Pugh S, Garden OJ, Cunningham D, Maughan T and Bridgewater $\mathrm{J}$ : Systemic chemotherapy with or without cetuximab in patients with resectable colorectal liver metastasis: the New EPOC randomised controlled trial. Lancet Oncol 15: 601-611, 2014.

19 Taieb J, Tabernero J, Mini E, Subtil F, Folprecht G, Van Laethem JL, Thaler J, Bridgewater J, Petersen LN, Blons H, Collette L, Van Cutsem E, Rougier P, Salazar R, Bedenne L, Emile JF, Laurent-Puig $\mathrm{P}$ and Lepage C: Oxaliplatin, fluorouracil, and leucovorin with or without cetuximab in patients with resected stage III colon cancer (PETACC-8): an open-label, randomised phase 3 trial. Lancet Oncol 15: 862-873, 2014.

20 Douillard JY, Oliner KS, Siena S, Tabernero J, Burkes R, Barugel M, Humblet Y, Bodoky G, Cunningham D, Jassem J, Rivera F, Kocákova I, Ruff P, Błasińska-Morawiec M, Šmakal M, Canon JL, Rother M, Williams R, Rong A, Wiezorek J, Sidhu $\mathrm{R}$ and Patterson SD: Panitumumab-FOLFOX4 treatment and RAS mutations in colorectal cancer. N Engl J Med 369: 1023$1034,2013$.

Received August 23, 2016

Revised September 16, 2016 Accepted September 21, 2016 